\title{
Locally resonant phononic woodpile: A wide band anomalous underwater acoustic absorbing material
}

\author{
Heng Jiang, ${ }^{1,2}$ Yuren Wang, ${ }^{2, a)}$ Milin Zhang, ${ }^{1}$ Yanping $\mathrm{Hu},{ }^{2}$ Ding Lan, ${ }^{2}$ Yinmin Zhang, ${ }^{2}$ \\ and Bingchen Wei ${ }^{2}$ \\ ${ }^{1}$ Key Laboratory of Superlight Materials and Surface Technology, Ministry of Education, \\ Harbin Engineering University, Harbin 150001, People's Republic of China \\ ${ }^{2}$ Key Laboratory of Microgravity Science, Institute of Mechanics, Chinese Academy of Sciences, \\ Beijing 100190, People's Republic of China
}

(Received 13 July 2009; accepted 12 August 2009; published online 9 September 2009)

\begin{abstract}
To meet the demand of modern acoustic absorbing material for which acoustic absorbing frequency region can be readily tailored, we introduced woodpile structure into locally resonant phononic crystal (LRPC) and fabricated an underwater acoustic absorbing material, which is called locally resonant phononic woodpile (LRPW). Experimental results show that LRPW has a strong capability of absorbing sound in a wide frequency range. Further theoretical research revealed that LRPC units and woodpile structure in LRPW play an important role in realization of wide band underwater strong acoustic absorption. (c) 2009 American Institute of Physics. [doi:10.1063/1.3216805]
\end{abstract}

The phononic crystal can control the propagation of acoustic wave by tailoring the phononic band gap that cannot be achieved by traditional acoustic materials. In the past decade, locally resonant phononic crystal (LRPC) has inspired great interest because it can exhibit an obvious phononic band-gap in the acoustic spectrum with crystal lattice constants two orders of magnitude smaller than the relevant sonic wavelength. ${ }^{1-4}$ Recent theoretical calculation has indicated that the maximum viscoelastic energy dissipation is generated at locally resonant frequency when considering viscoelastic deformation in LRPC..$^{5-7}$ It means that the LRPC can also be employed to expand the content of acoustic absorbing materials study. However, rare experimental studies are performed in this direction. Excellent underwater acoustic absorbing materials are urgently needed for their important applications in both military and commercial uses, such as sonar evasion by stealthy coating, underwater acoustic communication system. ${ }^{8-10}$ The underwater acoustic absorbing material is required to have a strong acoustic absorbance in a certain, relatively wide frequency region to satisfy various application purposes. This demand needs to develop acoustic absorbing material for which acoustic absorbing frequency region can be readily tailored. In this aspect, the LRPC material is a good candidate for material design. However, the LRPC has a strong absorption just at certain narrow frequency, which runs afoul of above requirement. To solve this conflict, we notice the pioneer works on the woodpile photonic crystal that can extend the band gap width of photonic crystal. ${ }^{11-14}$ In this paper, we introduced woodpile structure into the LRPC and developed a kind of underwater acoustic absorbing material, called "locally resonant phononic woodpile (LRPW)," which possesses underwater sound absorption property in a wide band of frequencies. The most striking difference between the LRPW and the LRPC is that locally resonant units in the LRPC distribute discretely in the polymer matrix, while those in LRPW have locally resonant woodpile structure stacked in sequence by LRPC units.

${ }^{a)}$ Electronic mail: yurenwang@imech.ac.cn.
When isolated two-dimensional units of the LRPC are connected to form three-dimensional woodpile structure, the number of locally resonant units is increased and the cooperative interaction between units is strengthened. The woodpile photonic crystal usually consists of layers of onedimensional rods with a stacking sequence that repeats itself every four layers. ${ }^{11-14}$ Two-dimensional LRPC was produced by periodically embedding the metallic rods coated with soft polymer layer into hard polymer matrix. ${ }^{15}$ Mimicking woodpile and LRPC structures, we coated a thin layer of soft polymer on woodpile skeleton and then filled the remaining space with hard polymer to make the LRPW.

It is easily known that a unique woodpile structure can only induce a finite-width band gap in the sound absorption spectrum. To further expand strong acoustic absorption frequency range, three kinds of woodpile structure layers containing steel rods with different diameters and square mesh with different areas were used to produce the LRPW. It is noted that these locally resonant woodpile structures are different from the traditional LRPC building blocks both in shape and in constitution. The enhancement on sound absorption was expected to occur in the LRPW.

The construction of locally resonant unit plays a key role in realization of locally resonant acoustic absorption. According to the LRPC formation mechanism, three kinds of materials with different elastic modulus are selected to produce the LRPW. They are steel rods with three different sizes, soft polyurethane (PU) and hard PU. Soft and hard PU are synthesized according to the method from literatures. ${ }^{16,17}$ In order to reduce the sound reflection on the surface of the LRPW, hard PU of characteristic impedance matching with water is chosen as viscoelastic coating. The synthesis route and structural morphology of the LRPW are shown in Fig. 1. Volume ratio of soft PU and steel rods is 3:1. The pulse tube method is often used for testing underwater sound reflection coefficients of materials in the field of water acoustics (Chinese National Standard GB/T 5266-2006), sound absorption coefficients of materials can be calculated by sound reflection coefficients. ${ }^{18}$ In this paper, underwater sound absorption coefficients of the LRPW are measured and calculated 


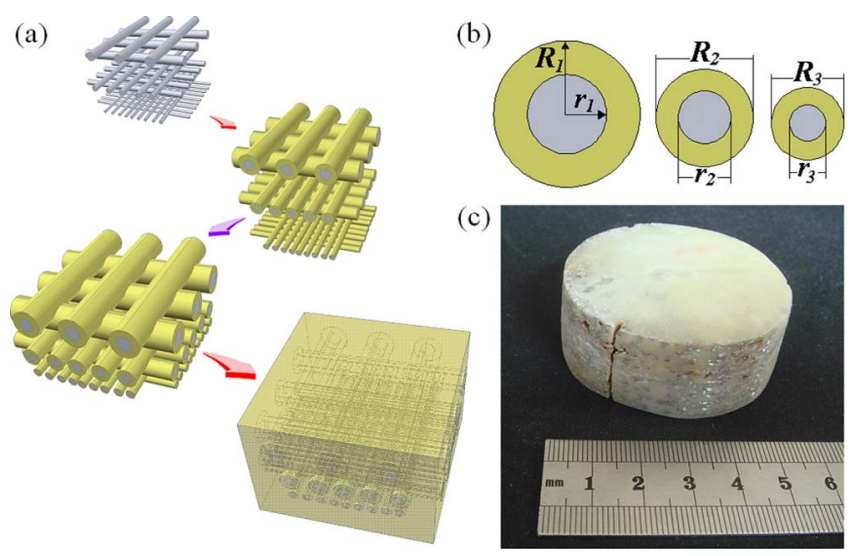

FIG. 1. (Color online) Schematic of synthesis route and optical image of LRPW. (a) Synthesis scheme. We produced three kinds of woodpile structure layers utilizing steel rods with three different diameters (steel square mesh sizes: $10 \times 10,5 \times 5$, and $2.5 \times 2.5 \mathrm{~mm}^{2}$ ). First, we coated a thin layer of soft PU on three kinds of woodpile structure based on the volume ratio of soft PU and steel rod. Steel woodpile layers coated with soft PU were stacked in sequence under uncured soft PU condition. After total drying of this soft PU layer, the uncured hard PU was filled into the remaining space. (b) Three kinds of steel rod coated with soft PU has been defined as $r_{1}$ $=0.55 \mathrm{~mm}, R_{1}=1.1 \mathrm{~mm}, r_{2}=0.35 \mathrm{~mm}, R_{2}=0.7 \mathrm{~mm}, r_{3}=0.25 \mathrm{~mm}$, and $R_{3}=0.5 \mathrm{~mm}$ according to the volume ratio of soft PU and steel rods. (c) The optical photo shows that sample is homogeneous solid material.

by the pulse tube system in the air back mode at Institute of Acoustics of Chinese Academy of Sciences. For comparison, all testing samples were fabricated with the same dimensions of $\Phi 56 \times 20 \mathrm{~mm}$.

Figure 2 shows sound absorption coefficients of the LRPW and other materials for comparison as a function of frequency. Absorption coefficients of LRPW are significantly higher than that of other materials. It can be seen that the LRPW has strong underwater acoustic absorbance with the average absorption coefficient over 0.8 in a wide frequency range, starting from $8 \mathrm{kHz}$. On the contrary, no sound absorption coefficients are higher than 0.8 for other materials in the tested frequency range. Aluminum foams and PU (component materials of the LRPW) are representatives of the traditional underwater acoustic absorbing materials. Their sound absorbing behaviors have been well studied in the literatures. ${ }^{19-22}$ The underwater acoustic absorption capabil-

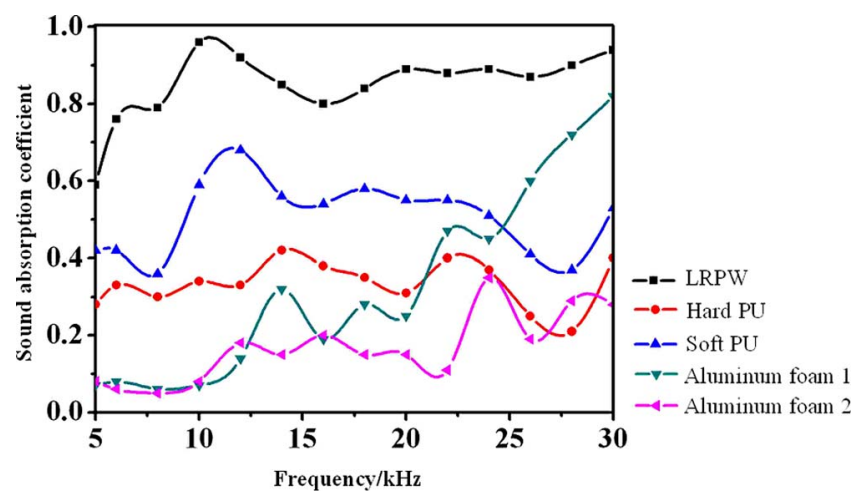

FIG. 2. (Color online) Comparison of underwater sound absorption coefficients for LRPW and other materials that were fabricated with the same dimensions in 5-30 kHz. Other materials included component materials of LRPW (soft PU and hard PU), aluminum foam 1 (the average pore size was $4 \mathrm{~mm}$ ) and aluminum foam 2 (the average pore size was $0.9 \mathrm{~mm}$ ). LRPW owns high average sound absorbing coefficient in $5-30 \mathrm{kHz}$, which could not be achieved by other materials.

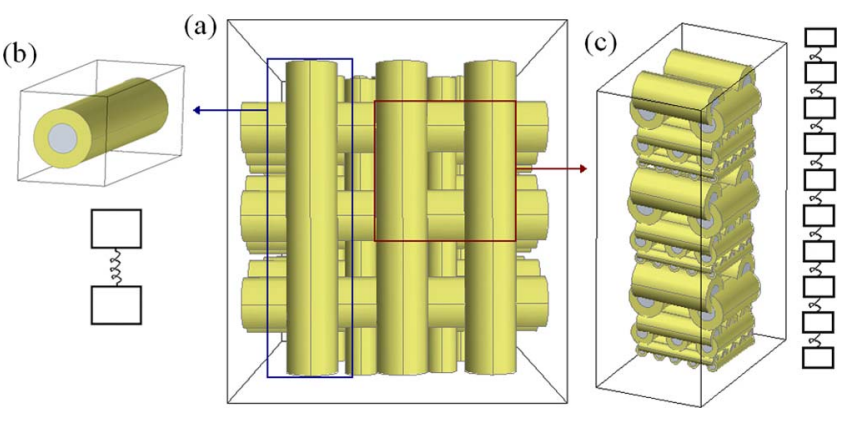

FIG. 3. (Color online) The model simplification process of LRPW. (a) Vertical view of $2 \mathrm{~cm}$ thick sample. Three sizes of steel woodpile layers coated with soft PU were stacked in sequence from perspective images. (b) In this picture, the space perspective effect shows that two-dimensional unit similar to that of LRPC exists in LRPW. According to the mass-spring model, two-dimensional unit of LRPC can be simplified to the double-oscillator model. (c) The right red solid square indicates a unit cell of LRPW. In this picture, the space perspective effect indicates a unit cell of $2 \mathrm{~cm}$ thick sample in the vertical direction. According to the mass-spring model, this unit cell can be simplified as ten vertical units connected by a fictitious spring.

ity of the LRPW is much better than that of traditional underwater acoustic absorbing materials at measured spectrum. The strong acoustic absorption characteristic of the LRPW is not originated by a certain component or simple linear superposition of acoustic absorption from its separate components. Therefore, it is reasonable to deduce that the combination of the LRPC units and woodpile structure is successful in achieving a wide band underwater strong acoustic absorbing material. Biot proposed a simple phenomenological theory of acoustic propagation in a porous, fluid filled, macroscopically homogeneous, and isotropic media. In his theory, solid and fluid were considered as two distinct interpenetrating "effective media." The acoustic absorbing behavior of aluminum foams can be explained by Biot theory. However, the acoustic absorbing phenomenon of the LRPW, which is a solid material, cannot be interpreted by Biot theory. Although two-dimensional locally resonant unit in the LRPW is similar to that of the LRPC, locally resonant woodpile structure of LRPW does not exist in LRPC. Therefore, the mechanism of wide band strong acoustic absorption in the LRPW needs to be further discussed although some relational theory of the LRPC can be utilized.

The lumped-mass method m $^{3,23-25}$ is a common and concise method to study band gap in the LRPC. It can simplify the complex calculation and effectively explain the mechanism. Therefore, to elucidate the physical effect of woodpile structures in physics, the lumped-mass method ${ }^{3,23-25}$ is employed to estimate possible sound resonant band gaps occurred in LRPW. The fully elastic scattering condition is used to simplify calculations. It is noted that the calculated band gaps can be considered to be the resonant absorbance frequencies if viscoelastic scattering is taken into account. The method can reveal the working principle of the LRPW, although it is relatively simple and rough. Figure 3(a) shows a schematic of woodpile structure in the LRPW while a cylindrical building unit is shown in Fig. 3(b). According to the lumped-mass method, ${ }^{3,23-25}$ a two-dimensional unit of the LRPC can be represented by a spring-mass model, as shown in Fig. 3(b). The calculated vibration frequency spectra are shown in Fig. 4(a). The gray hatched area indicated the phononic band gap which is induced by the two-dimensional 


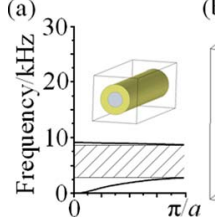

Wave vector $\mathrm{q}$

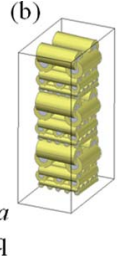

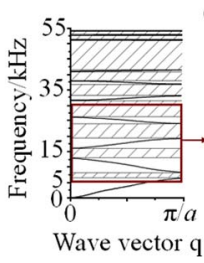

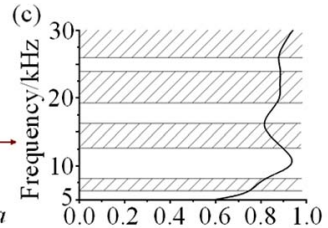

Wave vector $\mathrm{q}$ Sound absorption coefficient
FIG. 4. (Color online) Schematic of different locally resonant units and spectral characteristics. (a) Two-dimensional LRPC units only have relevant one band gap under full elastic scattering condition. (b) The LRPW unit of $2 \mathrm{~cm}$ thickness corresponds to nine band gaps of 6.3-54 kHz. (c) The measurement acoustic absorbing spectrum is mostly covered by band gaps spectrum of LRPW unit.

LRPC unit. It can be seen that the band gap just can cover a narrow frequency range form 2.8 to $8.8 \mathrm{kHz}$. This is a natural result because only vibration mode from individual twodimensional LRPC unit is considered in the calculation.

Locally resonant woodpile resonant unit of the LRPW is shown in the red square region of the right panel in Fig. 3(a). Therefore, the typical locally resonant woodpile structure unit of nine layers is shown in Fig. 3(c). According to the lumped-mass method, ${ }^{3,23-25}$ this LRPW unit can be simplified as ten vertical units connected by a virtual spring, as shown in the right panel of Fig. 3(c). The calculated vibration frequency spectra are shown in Fig. 4(b). An obvious character of spectra is that locally resonant woodpile band gaps covered different frequency ranges instead of a single band gap in Fig. 4(a). Spectra feature implied that the resonant sound reflection would happen in a more wide frequency range if locally resonant woodpile units were took into account. When the red solid square in Fig. 4(b) is enlarged, it can be seen that band gaps of locally resonant woodpile unit most cover the measurement acoustic absorbing spectrum of the LRPW from 5 to $30 \mathrm{kHz}$, as shown Fig. 4(c). The band gap widening effect is originated from the unique woodpile structure feature. Unlike the atomic crystal structures, the woodpile structure has an additional structure parameter referred to as filling factor. The band gap width for a woodpile structure is sensitive to the change of the filling factor. The frequency at which the lowest photon gap is centered is called the "midgap" frequency. According to the literature, a maximum gap to midgap ratio of $25 \%$ can be obtained at filling ratio $82 \% .^{11}$ Moreover, we introduced three kinds of locally resonant woodpile structures into the LRPW material that can further widen the applicable frequency range for the material. From Fig. 4(c) it can be deduced that the woodpile structure in the present samples enhanced locally resonant acoustic absorption capability and resulted in a broad strong absorption in the acoustic absorption curve. Therefore, this result gave a solid hint on the anomalous sound absorbing capability of the LRPW due to the combi- nation of LRPC units and woodpile structure.

In conclusion, we developed the LRPW material with high performance for underwater sound absorption, based on the theory of the LRPC and woodpile structure. Theoretical and experimental results revealed excellent sound absorption effect of the LRPW owing to the combination of LRPC structure units and woodpile structure of photonic crystal. The lumped-mass method is used to study the mechanism for strong sound absorbing of the LRPW. The concept of the LRPW gave a clue to the material design with excellent performance. The design concept presented in the study can also be extended to other functional materials.

We acknowledge project supported by the National Natural Science Foundation of China (Grant No 10832011) and the Knowledge Innovation Program of the Chinese Academy of Sciences (Grant No KJCX2-YW-L08).

${ }^{1}$ Z. Liu, X. Zhang, Y. Mao, Y. Y. Zhu, Z. Yang, C. T. Chan, and P. Sheng, Science 289, 1734 (2000).

${ }^{2}$ G. Wang, X. Wen, J. Wen, L. Shao, and Y. Liu, Phys. Rev. Lett. 93, 154302 (2004).

${ }^{3}$ M. Hirsekorn, Appl. Phys. Lett. 84, 3364 (2004).

${ }^{4}$ C. Goffaux, J. Sanchez-Dehesa, A. Levy Yeyati, Ph. Lambin, A. Khelif, J. O. Vasseur, and B. Djafari-Rouhani, Phys. Rev. Lett. 88, 225502 (2002).

${ }^{5}$ H. Zhao, Y. Liu, J. Wen, D. Yu, and X. Wen, Phys. Lett. A 367, 224 (2007).

${ }^{6}$ H. Zhao, Y. Liu, J. Wen, D. Yu, G. Wang, and X. Wen, Chin. Phys. Lett. 23, 2132 (2006).

${ }^{7}$ H. Zhao, Y. Liu, D. Yu, G. Wang, J. Wen, and X. Wen, J. Sound Vib. 303, 185 (2007).

${ }^{8}$ M. K. Hinders, B. A. Rhodes, and T. M. Fang, J. Sound Vib. 185, 219 (1995).

${ }^{9}$ D. Odell, K. Hertel, and C. Nelson, Oceans '02 MTS/IEEE 1, 266 (2002).

${ }^{10}$ M. Heinemann, A. Larraza, and K. B. Smith, J. Acoust. Soc. Am. 113, 3111 (2003).

${ }^{11}$ K. M. Ho, C. T. Chan, C. M. Soukoulis, R. Biswas, and M. Sigalas, Solid State Commun. 89, 413 (1994).

${ }^{12}$ S. Noda, K. Tomoda, N. Yamamoto, and A. Chutinan, Science 289, 604 (2000).

${ }^{13}$ S. Y. Lin, J. G. Fleming, D. L. Hetherington, B. K. Smith, R. Biswas, K. M. Ho, M. M. Sigalas, W. Zubrzycki, S. R. Kurtz, and J. Bur, Nature (London) 394, 251 (1998).

${ }^{14}$ G. M. Gratson, F. García-Santamaría, V. Lousse, M. Xu, S. Fan, J. A. Lewis, and P. V. Braun, Adv. Mater. 18, 461 (2006).

${ }^{15}$ C. Goffaux and J. Sanchez-Dehesa, Phys. Rev. B 67, 144301 (2003).

${ }^{16}$ S. L. Huang and J. Y. Lai, Eur. Polym. J. 33, 1563 (1997).

${ }^{17}$ S. L. Huang and J. Y. Lai, J. Membr. Sci. 105, 137 (1995).

${ }^{18}$ F. Han, G. Seiffert, Y. Zhao, and B. Gibbs, J. Phys. D 36, 294 (2003).

${ }^{19}$ P. H. Mott, C. M. Roland, and R. D. Corsaro, J. Acoust. Soc. Am. 111, 1782 (2002).

${ }^{20}$ M. A. Biot, J. Acoust. Soc. Am. 28, 168 (1956).

${ }^{21}$ M. A. Biot, J. Acoust. Soc. Am. 28, 179 (1956).

${ }^{22}$ D. L. Johnson and T. J. Plona, J. Acoust. Soc. Am. 72, 556 (1982).

${ }^{23}$ J. S. Jensen, J. Sound Vib. 266, 1053 (2003).

${ }^{24}$ G. Wang, J. Wen, Y. Liu, and X. Wen, Phys. Rev. B 69, 184302 (2004).

${ }^{25}$ G. Wang, J. Wen, and X. Wen, Phys. Rev. B 71, 104302 (2005). 